\title{
Hyperridge of triply excited states
}

\author{
Y. Komninos, M. Chrysos, and C. A. Nicolaides \\ Theoretical and Physical Chemistry Institute, National Hellenic Research Foundation, 48 Vassileos Constantinou Avenue, \\ Athens 11635, Greece
}

(Received 22 February 1988)

\begin{abstract}
We present a theory for the definition and the reliable calculation of correlated wave functions of a special class of triply excited states and for their rigorous geometrical analysis. This class is named the three-electron ionization ladder (THEIL) and refers to the simultaneous excitation of three electrons in valence states near threshold. Application to the Li THEIL of ${ }^{4} S^{\circ}$ symmetry reveals impressive localization properties. In analogy with a similar analysis of the "two-electron ionization ladder," which leads smoothly to the so-called "Wannier ridge" at the $E=0$ threshold, the present results suggest the existence of a "hyperridge" at $E=0$ with the following properties: (1) The ion core and the three electrons lie in a plane, (2) $\left|\mathbf{r}_{1}\right|=\left|\mathbf{r}_{2}\right|=\left|\mathbf{r}_{3}\right|$, and (3) $\theta_{12}=\theta_{23}=\theta_{31}$.
\end{abstract}

\section{INTRODUCTION}

In recent papers ${ }^{1-3}$ we have presented a quantitative theory of a special class of low- as well as high-lying doubly excited states (DES's) in polyelectronic atoms, which we have called the two-electron ionization ladder (TEIL). Analysis of their spectra and of their wave functions and the corresponding conditional probabilities as a function of the principal quantum number $n$ has led to the conclusion that the TEIL joins smoothly with the so-called Wannier state at the two-electron ionization threshold $(E=0) .^{4,5}$ According to the classical calculations and assumptions of Wannier, ${ }^{5}$ this state is characterized by the property $\mathbf{r}_{1}=-\mathbf{r}_{2}$ at $E=0$, an important property which has been called the Wannier ridge. ${ }^{4}$

A rigorous quantum-mechanical many-electron theory and calculation of the two-electron continuous spectrum near threshold in the presence of a core is not yet available. However, important insight can be obtained via alternative routes of investigation. These refer to the possibility of understanding the very-high- $n$ and threshold regions by joining smoothly with information from wave functions and observables obtained accurately for the TEIL below.

In this paper we turn our attention to the physics of low- as well as high-lying triply excited states (TES's). Although their experimental observation and study is not easy yet, ${ }^{6}$ their quantitative understanding, especially as regards their electronic structure, their properties, and their complete fragmentation dynamics, is an intriguing theoretical problem, whose complexity exceeds by far the corresponding one of the two-electron problem. ${ }^{7}$ Our aim has been to develop a theory in such a way as to be able to obtain first principles and accurate information as regards the spectrum and the wave functions of real atomic systems and then extract from this information additional properties. Although this aim has many facets, the related fundamental question has been: What are the geometrical and dynamical properties of the three-electron threshold ionization state? As in the case of the two electrons, this question addresses the very difficult problem of treating electronic correlations close to threshold. This implies that the theory should be applicable beyond the very-low- $n$ region. ${ }^{8}$

\section{THEORY AND CALCULATIONS}

Responding to the question stated above, we expect, by conceptual analogy to the two-electron Wannier state and by symmetry, that, at $E=0$, the simultaneous ejection of the three electrons will occur in a plane which includes the remaining ion and with the position-space property

$$
\left|\mathbf{r}_{1}\right|=\left|\mathbf{r}_{2}\right|=\left|\mathbf{r}_{3}\right|
$$

and

$$
\theta_{12}=\theta_{23}=\theta_{31} \text {. }
$$

This geometry defines the hyperridge of the three-electron excitation and ionization problem.

Is the hyperridge geometry realizable? The theory and calculations of this work yield a positive answer.

Our first conceptual and computational steps are based on the main inputs and results of the TEIL theory. ${ }^{1-3}$ These are as follows.

(1) For each manifold of valence DES's characterized by $n_{1}=n_{2}$, the TEIL state corresponds to the state of lowest energy (electron correlation criterion).

(2) The DES's of polyelectronic atoms are treated quantitatively in a state-specific manner. ${ }^{1,9}$ The computation of the appropriate zeroth-order vector is carried out within multiconfiguration Hartree-Fock (MCHF) theory with valence configurations characterized by $n_{1}=n_{2}$ only (zeroth-order, concerted motion criterion; in this way, angular as well as a large portion of radial correlation are computed systematically).

(3) Given the MCHF solution, the remaining correlation is obtained variationally. It is found that the proximity of the result of the zeroth-order calculation to that of the fully correlated one improves as $n$ increases.

(4) For relatively high $n$, the only available experimental spectrum is that of Buckman and co-workers ${ }^{10}$ in 
$\mathrm{He}^{-}$. Our theory agrees with it. ${ }^{2,10}$ The geometry of the TEIL states is derived via appropriate definitions and calculations of expectation values and probability densities, and can be used to deduce ${ }^{3}$ the analytic formula for the TEIL spectrum in small atoms,

$$
E_{n}(\mathrm{TEIL})=A \frac{n(n-1)}{r_{n}^{2}},
$$

where $A$ is a constant characterizing the system and $r_{n} \equiv\left\langle r_{1}\right\rangle_{n}=\left\langle r_{2}\right\rangle_{n}$, computed from first principles. ${ }^{1-3}$

The aforementioned questions and facts have led us to the definition of the three-electron ionization ladder (THEIL) and to a corresponding many-electron theory which is analogous, but more complex than that of the TEIL. The three-electron system which was studied is the triply excited $\mathrm{Li}$ coupled to a ${ }^{4} S^{\circ}$ symmetry and parity. The configuration corresponding to the lowest state of this symmetry is the $2 p^{3}{ }^{\circ} S^{\circ}$. This state was examined more than ten years ago ${ }^{11,12}$ in relation to the problem of determining the number and the type of excited discrete states in negative ions (e.g., Refs. 12 and 13). It was then found that $\mathrm{H}^{-}-$is unbound but that $\mathrm{He}^{-{ }^{4}} \mathrm{~S}^{\circ}$ is bound nonrelativistically due to electron correlation. ${ }^{11,14}$

In order to proceed with the analysis which is related to the question of the hyperridge geometry, we first had to obtain THEIL wave functions. The following calculations were carried out.

(1) The exact wave functions (localized part only) are written as

$$
T_{n}=T_{n}^{0}+X_{n}
$$

where $T_{n}^{0}$ is the MCHF zeroth-order solution at each shell $n$. The $T_{n}^{0}$ contain angular as well as a large part of radial correlation and their characteristics are very close to those of $T_{n}$. Thus we confined ourselves to the calculation of $T_{n}^{0}$ only, since their quality is perfectly adequate for our purposes.

(2) We computed up to $n=5$. It was deemed unnecessary to go any higher since, as $n$ increases, the calculations become more difficult and expensive and since the information which has been obtained from these wave functions is sufficient to draw our conclusions (see below). As an example, the $n=4 T_{n}^{0}$ of ${ }^{4} S^{\circ}$ symmetry is given by

$$
\begin{aligned}
T_{4}^{0}= & 0.788\left(4 p^{3}\right)+0.557\left(4 p 4 d^{2}\right)+0.257\left(4 f 4 d^{2}\right) \\
& +0.053\left(4 f^{3}\right)-0.002\left(4 p 4 f^{2}\right) .
\end{aligned}
$$

We note that configurations which represent open channels are excluded from the TEIL or THEIL calculations, since they cannot contribute significantly to the problem at hand. In the region of interest ( $\left.r_{1} \approx r_{2} \approx r_{3}=\langle r\rangle\right)$ the corresponding core orbitals are of short range and do not have a substantial - if at allamplitude.

Having defined and calculated the $T_{n}^{0}$ wave functions, the following analysis was developed and applied. Our goal is to deduce the geometrical behavior of these threeelectron excited states as a function of $n$. Two properties are relevant. The first is the average radii of the electrons. The second is the average angle between pairs of
TABLE I. Values of $\langle r\rangle_{n}$ and $\left\langle\theta_{12}\right\rangle_{n}$ for $n=3,4,5$, calculated from the $T_{n}^{0}$ wave functions of this work. As $n$ increases, $\left\langle\theta_{12}\right\rangle_{n}$ tends to $120^{\circ}$.

\begin{tabular}{ccc}
\hline$n$ & $r_{n}($ a.u. $)$ & $\left\langle\theta_{12}\right\rangle_{n}(\mathrm{deg})$ \\
\hline 3 & 5.0 & 99.6 \\
4 & 9.0 & 105.2 \\
5 & 14.0 & 110.1 \\
\hline
\end{tabular}

electrons with respect to the nucleus.

The first property is obtained immediately from the expectation values of $r$ and from the fact that all the MCHF orbitals in $T_{n}^{0}$ have nearly the same $\langle r\rangle_{n l}$. Table I shows the THEIL radii and Fig. 1 shows the graphs of the orbitals in $T_{4}^{0}$ as a function of $r$.

The second property is derived by calculating the expectation value $\left\langle\cos \theta_{12}\right\rangle$ and by calculating and plotting the density $\rho\left(\hat{r}_{1}, \hat{r}_{2}\right)$ [see Eq. (9)] for an arbitrary position of the third electron. The related physics and algebra are as follows.

First, we integrate the radial part out of the probability density function. Given that the $T_{n}^{0}$ contain only MCHF configurations with $n_{1}=n_{2}=n_{3}=n$, no cross terms appear and the radial integral is normalized to unity. If $\Psi\left(\hat{r}_{1}, \hat{r}_{2}, \hat{r}_{3}\right)$ is the angular part of the total wave function,

$$
\begin{array}{rl}
\left\langle\cos \theta_{12}\right\rangle=\int_{\Omega_{1}} \int_{\Omega_{2}} \int_{\Omega_{3}} & d \Omega_{1} d \Omega_{2} d \Omega_{3} \Psi^{*}\left(\hat{r}_{1}, \hat{r}_{2}, \hat{r}_{3}\right) \cos \theta_{12} \\
\times \Psi\left(\hat{r}_{1}, \hat{r}_{2}, \hat{r}_{3}\right),
\end{array}
$$

where $\hat{r}_{i}(i=1,2,3)$ represents the unit position vector of the $i$ th electron with respect to the nucleus.

In order to carry out the triple integral (5), we construct the $\Psi$ function of ${ }^{4} S^{\circ}$ symmetry by coupling the three electrons sequentially,

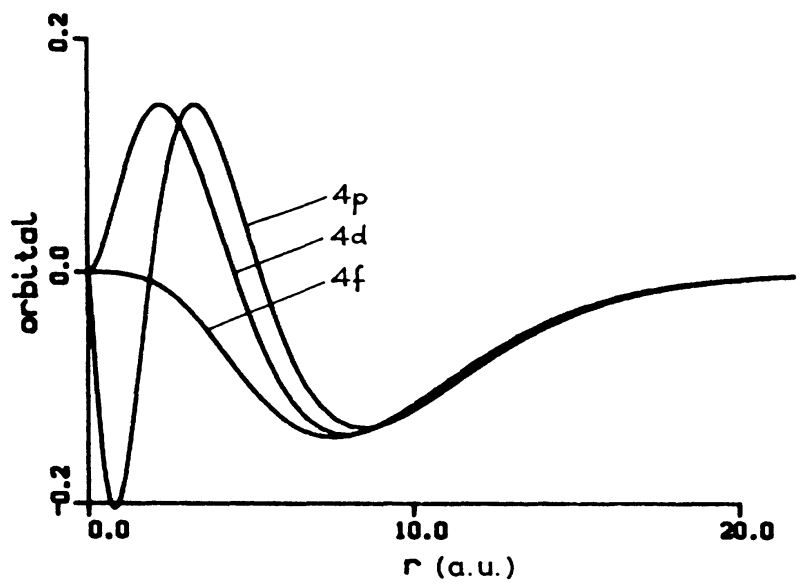

FIG. 1. Plots of the MCHF orbitals for $T_{4}^{0}(n=4)$. The average values are $\langle r\rangle_{4 p}=9.0$ a.u., $\langle r\rangle_{4 d}=8.9$ a.u., and $\langle r\rangle_{4 f}=9.0$ a.u. The extreme closeness of these values implies that calculating an $\langle r\rangle_{4}$ from the total wave function is meaningful. 
$\Psi\left(\widehat{r}_{1}, \widehat{r}_{2}, \hat{r}_{3}\right)=\sum_{l_{1}, l_{2}, l_{3}, L} C_{l_{1} l_{2} l_{2}} D\left(l_{1}, l_{2}, l_{3}\right) \sum_{m_{3}, M}\left\langle l_{3} L m_{3} M \mid 00\right\rangle Y_{l_{3}}^{m_{3}}\left(\hat{r}_{3}\right) \sum_{m_{1}, m_{2}}\left\langle l_{1} l_{2} m_{1} m_{2} \mid L M\right\rangle Y_{l_{1}}^{m_{1}}\left(\widehat{r}_{1}\right) Y_{l_{2}}^{m_{2}}\left(\widehat{r}_{2}\right)$,

and then,

$\Psi\left(\widehat{r}_{1}, \hat{r}_{2}, \hat{r}_{3}\right)=\sum_{l_{1}, l_{2}, l_{3}} C_{l_{1}, l_{2}, l_{3}} D\left(l_{1}, l_{2}, l_{3}\right) \sum_{m_{1}, m_{2}, m_{3}}(-1)^{l_{1}-l_{2}+l_{3}}\left(\begin{array}{ccc}l_{1} & l_{1} & l_{3} \\ m_{1} & m_{2} & m_{3}\end{array}\right) Y_{l_{1}}^{m_{1}}\left(\widehat{r}_{1}\right) Y_{l_{2}}^{m_{2}}\left(\hat{r}_{2}\right) Y_{l_{3}}^{m_{3}}\left(\widehat{r}_{3}\right)$,

where $C_{l_{1} l_{2} l_{3}}$ are the $T_{n}^{0}$ configuration coefficients and $D\left(l_{1}, l_{2}, l_{3}\right)$ is a normalizing factor which depends on the form of the configurations:

$D\left(l_{1}, l_{2}, l_{3}\right)= \begin{cases}1 \text { for } l_{1}=l_{2}=l_{3} \\ \frac{1}{\sqrt{3}} \text { for } l_{1}=l_{2} \neq l_{3} \\ \frac{1}{\sqrt{6}} \text { for } l_{1} \neq l_{2} \neq l_{3} \neq l_{1} .\end{cases}$

Using expression (6b) the probability density of the angular part of $T_{n}^{0}$ is written as

$$
\begin{aligned}
& \widetilde{\rho}\left(\widehat{r}_{1}, \hat{r}_{2,}, \hat{r}_{3}\right)=\sum_{l_{1}^{\prime}, l_{2}^{\prime}, l_{3}^{\prime} l_{1}, l_{2}, l_{3}} C_{l_{1}^{\prime} l_{2}^{\prime} l_{3}^{\prime}} C_{l_{1} l_{2} l_{3}} D\left(l_{1}^{\prime}, l_{2}^{\prime}, l_{3}^{\prime}\right) D\left(l_{1}, l_{2}, l_{3}\right) \\
& \times \sum_{m_{1}^{\prime}, m_{2}^{\prime}, m_{3}^{\prime}} \sum_{m_{1}, m_{2}, m_{3}}(-1)^{l_{1}^{\prime}-l_{2}^{\prime}+l_{3}^{\prime}}(-1)^{l_{1}-l_{2}+l_{3}}\left(\begin{array}{ccc}
l_{1}^{\prime} & l_{2}^{\prime} & l_{3}^{\prime} \\
m_{1}^{\prime} & m_{2}^{\prime} & m_{3}^{\prime}
\end{array}\right]\left[\begin{array}{ccc}
l_{1} & l_{2} & l_{3} \\
m_{1} & m_{2} & m_{3}
\end{array}\right)
\end{aligned}
$$

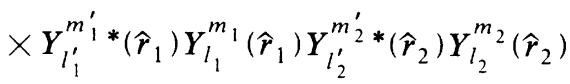

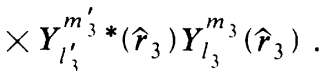

We now single out one of the electrons by integrating over its angular space $\Omega_{3}$. Thus

$$
\begin{aligned}
& \rho\left(\widehat{r}_{1}, \widehat{r}_{2}\right) \equiv \int d \Omega_{3} \widetilde{\rho}\left(\widehat{r}_{1}, \widehat{r}_{2}, \widehat{r}_{3}\right) \\
& =\sum_{l_{3}} \sum_{m_{3}} \sum_{l_{1}^{\prime}, l_{2}^{\prime} l_{1}, l_{2}} C_{l_{1}^{\prime} l_{2}^{\prime} l_{3}} C_{l_{1} l_{2} l_{3}} D\left(l_{1}^{\prime}, l_{2}^{\prime}, l_{3}\right) D\left(l_{1}, l_{2}, l_{3}\right) \\
& \times \sum_{m_{1}^{\prime}, m_{2}^{\prime}} \sum_{m_{1}, m_{2}}(-1)^{l_{1}^{\prime}-l_{2}^{\prime}}(-1)^{l_{1}-l_{2}}\left(\begin{array}{ccc}
l_{1}^{\prime} & l_{2}^{\prime} & l_{3}^{\prime} \\
m_{1}^{\prime} & m_{2}^{\prime} & m_{3}^{\prime}
\end{array}\right)\left(\begin{array}{ccc}
l_{1} & l_{2} & l_{3} \\
m_{1} & m_{2} & m_{3}
\end{array}\right) \\
& \times Y_{l_{1}^{\prime}}^{m_{1}^{\prime}} *\left(\widehat{r}_{1}\right) Y_{l_{1}}^{m_{1}}\left(\hat{r}_{1}\right) Y_{l_{2}^{\prime}}^{m_{2}^{\prime} *}\left(\widehat{r}_{2}\right) Y_{l_{2}}^{m_{2}}\left(\widehat{r}_{2}\right),
\end{aligned}
$$

where only terms $l_{3}=l_{3}^{\prime}$ and $m_{3}=m_{3}^{\prime}$ have survived.

Upon execution of a rather tedious angular momentum algebra, Eq. (8) reduces to

$$
\begin{array}{r}
\rho\left(\hat{r}_{1}, \widehat{r}_{2}\right)=\sum_{l_{3}} \sum_{l_{1}^{\prime}, l_{2}^{\prime} l_{1}, l_{2}} C_{l_{1}^{\prime} l_{2}^{\prime} l_{3}} C_{l_{1} l_{2} l_{3}} D\left(l_{1}^{\prime}, l_{2}^{\prime}, l_{3}\right) D\left(l_{1}, l_{2}, l_{3}\right)\left[\left(2 l_{1}^{\prime}+1\right)\left(2 l_{2}^{\prime}+1\right)\left(2 l_{1}+1\right)\left(2 l_{2}+1\right)\right]^{1 / 2} \\
\times \sum_{k=k_{\min }}^{k_{\max }}(-1)^{l_{3}-k} \frac{2 k+1}{2} P_{k}\left(\cos \theta_{12}\right) \\
{\left[\begin{array}{ccc}
l_{1} & l_{1}^{\prime} & k \\
0 & 0 & 0
\end{array}\right]\left[\begin{array}{ccc}
l_{1} & l_{2}^{\prime} & k \\
0 & 0 & 0
\end{array}\right]\left\{\begin{array}{lll}
l_{1} & k & l_{1}^{\prime} \\
l_{2}^{\prime} & l_{3} & l_{2}
\end{array}\right\},}
\end{array}
$$

where

$$
\begin{aligned}
& k_{\min }=\max \left(\left|l_{1}-l_{1}^{\prime}\right|,\left|l_{2}-l_{2}^{\prime}\right|\right), \\
& k_{\max }=\min \left(l_{1}+l_{2}^{\prime}, l_{2}+l_{2}^{\prime}\right) .
\end{aligned}
$$

A formula similar to Eq. (9) has been derived before for the reduced density of a doubly excited state. ${ }^{15}$ Finally, using Eq. (9), we obtain

$$
\begin{aligned}
\left\langle\cos \theta_{12}\right\rangle= & \int_{-1}^{1} d\left(\cos \theta_{12} \rho\left(\widehat{r}_{1}, \widehat{r}_{2}\right) \cos \theta_{12}\right. \\
= & \sum_{l_{3}} \sum_{l_{1}^{\prime}, l_{2}^{\prime} l_{1}, l_{2}} C_{l_{1}^{\prime} l_{2}^{\prime} l_{3}} C_{l_{1} l_{2} l_{3}} D\left(l_{1}^{\prime}, l_{2}^{\prime}, l_{3}\right) D\left(l_{1}, l_{2}, l_{3}\right)(-1)^{l_{3}-1} \\
& \times\left[\left(2 l_{1}^{\prime}+1\right)\left(2 l_{2}^{\prime}+1\right)\left(2 l_{1}+1\right)\left(2 l_{2}+1\right)\right]^{1 / 2}\left[\begin{array}{ccc}
l_{1} & l_{1}^{\prime} & 1 \\
0 & 0 & 0
\end{array}\right]\left[\begin{array}{ccc}
1_{2} & l_{2}^{\prime} & 1 \\
0 & 0 & 0
\end{array}\right]\left\{\begin{array}{lll}
l_{1} & 1 & l_{1}^{\prime} \\
l_{2}^{\prime} & l_{3} & l_{2}
\end{array}\right\},
\end{aligned}
$$




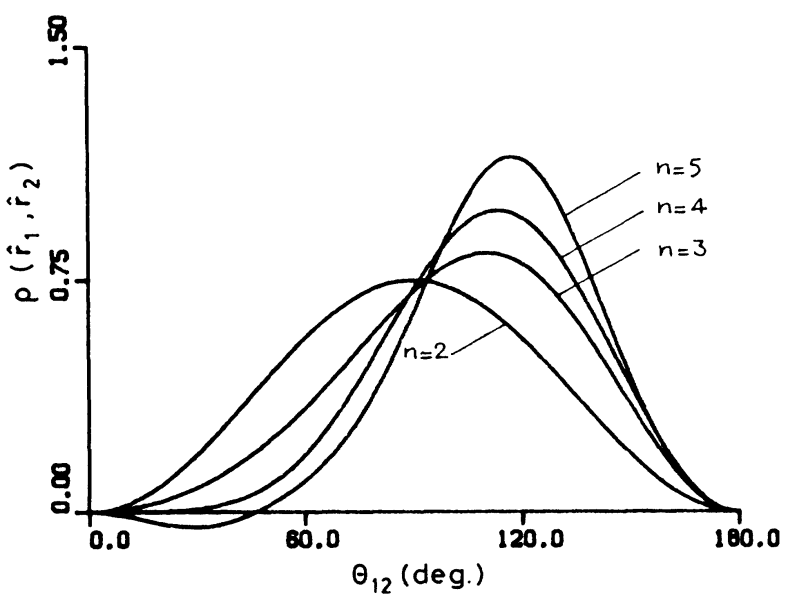

FIG. 2. Plot of $\rho\left(\hat{r}_{1}, \hat{r}_{2}\right)$ [Eq. (9)] as a function of the angle $\theta_{12}$. For $n=2$, i.e., for the single configuration $2 p^{34} S^{\circ}$, the distribution is symmetric around $90^{\circ}$. As $n$ increaes, the curve becomes increasingly skew-symmetric, while $\left\langle\theta_{12}\right\rangle$ tends to $120^{\circ}$ at the threshold. The small negative part for $n=5$ is due to numerical inaccuracy.

where $l_{1}^{\prime}, l_{2}^{\prime}, l_{1}, l_{2}$, are such that

$\max \left(\left|l_{1}-l_{2}^{\prime}\right|,\left|l_{2}-l_{2}^{\prime}\right|\right) \leq 1 \leq \min \left(l_{1}+l_{1}^{\prime}, l_{2}+l_{2}^{\prime}\right)$.

\section{RESULTS AND CONCLUSION}

The numerical implementation of Eqs. (9) and (10) reveals an impressive geometrical property. This is presented in Table I, for the values of $r_{n}$ and $\left\langle\cos \theta_{12}\right\rangle_{n}$ $(n=3,4,5)$, and in Figs. 2 and 3. Figure 2 shows the plot of $\rho\left(\hat{r}_{1}, \widehat{r}_{2}\right)$ as a function of angle and Fig. 3 shows geometrical "pictures" of the valence electrons as a function of level of excitation. As $n \rightarrow \infty$, we expect the realization of the hyperridge geometry of Eq. (1).

In conclusion, the theory and calculations of this paper offer conceptual as well as quantitative insight into the physics of simultaneous excitation and threshold ionization of three valence electrons. We point out that the validity of our analysis depends on the fact that it is angular

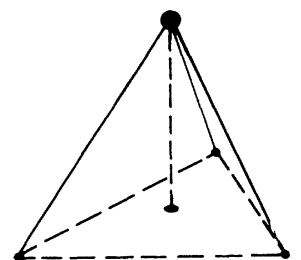

(a)

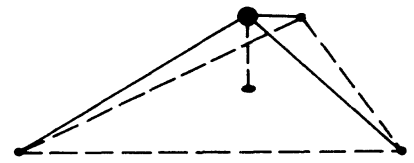

(b)

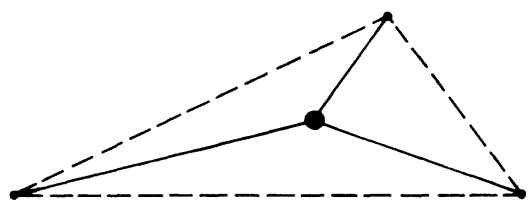

(c)

FIG. 3. Geometrical "pictures" of the THEIL ${ }^{4} S^{\circ}$ states as a function of $n$, based on the results of the present theory. (a) Low $n$. (b) Higher $n$. (c) $n \rightarrow \infty$. As $n$ increases, the pyramid opens up and flattens out, tending toward the hyperridge geometry of (c), where the nucleus and the three electrons lie in the plane with $\theta_{12}=\theta_{23}=\theta_{31}$, and $\left|\mathbf{r}_{1}\right|=\left|\mathbf{r}_{2}\right|=\left|\mathbf{r}_{3}\right|$.

correlations which must be computed accurately for such properties. Thus the trend for hyperridge shows up reliably already at $n=5$. Nothing new would be gained in this respect by doing calculations at higher $n$. Given the generality of the theory and its computational advantages, it is reasonable to expect, in future work, the prediction of accurate spectra of experimentally verifiable THEIL's and, possibly, of formulas analogous to Eq. (2).
'Y. Komninos and C. A. Nicolaides, J. Phys. B 19, 1701 (1986); C. A. Nicolaides and Y. Komninos, Phys. Rev. A 35, 999 (1987).

${ }^{2}$ Y. Komninos, M. Chrysos, and C. A. Nicolaides, J. Phys. B 20, L791 (1987).

${ }^{3}$ C. A. Nicolaides, M. Chrysos, and Y. Komninos, J. Phys. B 21, L73 (1988).

${ }^{4}$ U. Fano, Rep. Prog. Phys. 46, 97 (1983); A. R. P. Rau, J. Phys. (Paris) Coll. 43, 211 (1982).

${ }^{5}$ G. H. Wannier, Phys. Rev. 90, 817 (1953); 100, 1180 (1955).

${ }^{6}$ See C. A. Nicolaides and D. R. Beck, J. Chem. Phys. 66, 1982 (1977), for a theoretical prediction of the photoabsorption os- cillator strengths to low-lying triply excited states of ${ }^{2} P^{\circ}$ symmetry in $\mathrm{Li}$.

${ }^{7}$ For example, see the recent paper by S. Watanabe and C. D. Lin, Phys. Rev. A 36, 511 (1987), who carried out a model calculaton on low-lying triply excited states from a molecular point of view.

${ }^{8}$ In the case of a DES, the knowledge obtained from the study of the low-lying shells, to which other theories have been limited, is not conclusive for properties depending on radial correlations-even if it is quantitative.

${ }^{9}$ Y. Komninos, N. Makri, and C. A. Nicolaides, Z. Phys. D 2, 105 (1986). 
10S. J. Buckman and D. J. Newman, J. Phys. B 20, L711 (1987); S. J. Buckman, P. Hammond, F. H. Read, and G. C. King, ibid. 16, 4039 (1983).

${ }^{11}$ D. R. Beck and C. A. Nicolaides, Chem. Phys. Lett. 59, 525 (1978).

${ }^{12}$ C. A. Nicolaides and Y. Komninos, Chem. Phys. Lett. 80, 463 (1981); C. A. Nicolaides, Y. Komninos, and D. R. Beck, Phys. Rev. A 24, 1103 (1981).

${ }^{13}$ G. Aspromallis, C. A. Nicolaides, and Y. Komninos, J. Phys. B 18, L545 (1985); C. F. Bunge, M. Galan, R. Jauregui, and
A. V. Bunge, Nucl. Instrum. Methods 202, 299 (1983); D. R. Beck, Phys. Rev. A 30, 3305 (1984); C. Froese-Fischer, J. B. Lagowski, and S. H. Vosko, Phys. Rev. Lett. 59, 2263 (1987); D. R. Beck, Phys. Rev. A 37, 1847 (1988).

${ }^{14} \mathrm{The} \mathrm{He}^{-{ }^{4}} S^{\circ}$ state has been shown to have a particular spectrum and decay dynamics which are suitable for the construction of an atomic "excimer" laser of short wavelength. [See Ref. 12 and C. A. Nicolaides, IEEE J. Quantum. Electron. QE-19, 1781 (1983).]

${ }^{15}$ G. S. Ezra and R. S. Berry, Phys. Rev. A 25, 1513 (1982). 